\title{
Wavelet Analysis of Shower Track Distribution in High-Energy Nucleus-Nucleus Collisions
}

\author{
Provash Mali, ${ }^{1}$ Soumya Sarkar, ${ }^{1}$ Amitabha Mukhopadhyay, ${ }^{1}$ and Gurmukh Singh ${ }^{2}$ \\ ${ }^{1}$ Department of Physics, University of North Bengal, Darjeeling 734013, India \\ ${ }^{2}$ Department of Computer and Information Science, SUNY at Fredonia, Fredonia, NY 14063, USA
}

Correspondence should be addressed to Amitabha Mukhopadhyay; amitabha_62@rediffmail.com

Received 19 June 2013; Accepted 7 November 2013

Academic Editor: Sakina Fakhraddin

Copyright (c) 2013 Provash Mali et al. This is an open access article distributed under the Creative Commons Attribution License, which permits unrestricted use, distribution, and reproduction in any medium, provided the original work is properly cited.

\begin{abstract}
A continuous wavelet analysis is performed for pattern recognition of charged particle emission data in ${ }^{28} \mathrm{Si}-\mathrm{Ag} / \mathrm{Br}$ interaction at $14.5 \mathrm{~A} \mathrm{GeV}$ and in ${ }^{32} \mathrm{~S}-\mathrm{Ag} / \mathrm{Br}$ interaction at $200 \mathrm{~A} \mathrm{GeV}$. Making use of the event-wise local maxima present in the scalograms, we try to identify the collective behavior in multiparticle production, if there is any. For the first time, the wavelet results are compared with a model prediction based on the ultrarelativistic quantum molecular dynamics (UrQMD), where we adopt a charge reassignment algorithm to modify the UrQMD events to mimic the Bose-Einstein type of correlation among identical mesons-a feature known to be the most dominating factor responsible for local cluster formation. Statistically significant deviations between the experiment and the simulation are interpreted in terms of nontrivial dynamics of multiparticle production.
\end{abstract}

\section{Introduction}

The primary objective of studying high-energy heavy-ion interactions is to compress and heat up the nuclear matter beyond the critical values of certain thermodynamic parameters in such a way that the boundaries of individual nucleons melt down to form a thermally and chemically equilibrated color deconfined state of quark-gluon plasma (QGP) [1-3]. As the collision process evolves in space-time, such an exotic state, if formed, subsequently expands and cools down to undergo a reverse transition to the usual hadronic state. In high-energy physics, the process is known as multiparticle production. Each nucleus-nucleus $(A B)$ event has its own collision history that ultimately leads to large local fluctuations in the final state particle densities, apparently lacking any definite pattern. In different events, dense clusters of particles are formed at different locations and at different scales of phase-space variables. It is therefore, necessary to formulate a technique that can examine these clusters on an event by event basis. Often, the fluctuations are so large that they can not be explained simply in terms of statistical reasons. It is all very likely that nonstatistical (dynamical) components are present as well, but they are contaminated with trivial noise. With the help of suitable data analysis techniques, it is possible to filter out the genuine clusters of produced particles that in many high-energy interactions are found to scale selfsimilarly with the phase-space resolution size, approximately following a power law [4]. Global analysis techniques such as the scaled factorial moment method $[5,6]$, the frequency moment method $[7,8]$, and the " $S$-parameter" method [9] have extensively been used to characterize the particle correlations, and efforts are made to interpret the results in the framework of various mechanisms that are mostly speculative in nature. Formation of the QCD parton shower cascade [10], formation of the disoriented chiral condensate [11, 12], and collective phenomena like the emission of Cherenkov gluons and/or Mach shock wave in the nuclear/partonic medium $[13,14]$ are examples of some such speculative measures.

The wavelet analysis technique has found its application in many branches of physics $[15,16]$. It is capable of revealing the local properties of particle distributions in individual events and at different scales. The technique is, therefore, very appropriate for pattern recognition in multiparticle distribution. In the present paper, we report some results on the wavelet analysis of the angular distribution of shower tracks that are caused by the singly charged produced particles 
moving with relativistic speed. Our data samples comprise ${ }^{28} \mathrm{Si}-\mathrm{Ag} / \mathrm{Br}$ events at an incident energy of $14.5 \mathrm{~A} \mathrm{GeV}$ and ${ }^{32} \mathrm{~S}-\mathrm{Ag} / \mathrm{Br}$ events at an incident energy of $200 \mathrm{~A} \mathrm{GeV}$. Nuclear emulsion technique has been used to collect the experimental data. Several works on the wavelet analysis of multiparticle production at $E_{\text {lab }}=10-10^{3} \mathrm{GeV} /$ nucleon have so far been reported [17-21]. These works suffer from a common drawback in the sense that there has hardly been any comparison between the experiment and a proper simulation on $A B$ interaction. It is, therefore, difficult to conclude whether the experimental observations are significant or they are consequences of mere statistical artifacts. We compare our results with the predictions of a microscopic transport model based on the ultrarelativistic quantum molecular dynamics (UrQMD) $[22,23]$. It may also be noted that the UrQMD code does not incorporate the Bose-Einstein correlation (BEC) among the identical mesons, a phenomenon considered to be the most dominant factor behind particle cluster formation. Therefore, keeping the phase-space distribution of the produced particles (mostly $\pi$-mesons) unaltered, we implement a charge reassignment algorithm [24-26] to each UrQMD generated event and thereby try to mimic the BEC into simulation. Any discrepancy between the experiment and the simulation should now be recognized as a genuine collective behavior of the final state particle emission, which has to be interpreted in terms of nontrivial dynamics. Thus, the present analysis on one hand allows us to compare experiments induced by very close projectile masses, while the corresponding $E_{\mathrm{lab}}$ values differ by an order of magnitude; on the other hand, it provides an opportunity to compare the experiment with such simulated data where the known dominant source(s) of cluster formation is taken into account. Our paper is organized according to the following sequence: in Section 2, we briefly describe the experiment and the gross characteristics of the data samples used in the paper; in Section 3, we summarily discuss the basic aspects of the UrQMD model and explain the charge reassignment algorithm; in Section 4, without claiming any originality we outline the method of wavelet analysis; in Section 5, we discuss our results-experimental as well as simulated; and in Section 6, we conclude with a critical assessment of these results.

\section{Experiment}

Ilford G5 nuclear photoemulsion pellicles of size $16 \mathrm{~cm} \times$ $10 \mathrm{~cm} \times 600 \mu \mathrm{m}$ were horizontally irradiated with ${ }^{28} \mathrm{Si}$ beam at an incident energy of $14.5 \mathrm{~A} \mathrm{GeV}$ from the alternating gradient synchrotron (AGS) of the Brookhaven National Laboratory (BNL). Similarly pellicles of size $18 \mathrm{~cm} \times 7 \mathrm{~cm} \times$ $600 \mu \mathrm{m}$ were irradiated with $\mathrm{a}^{32} \mathrm{~S}$ beam at an incident energy of $200 \mathrm{~A} \mathrm{GeV}$ from the super proton synchrotron (SPS) at CERN. The primary interactions (also called events/stars) within the emulsion plates are found by following individual projectile tracks, that is, tracks caused by the ${ }^{28} \mathrm{Si}$ and ${ }^{32} \mathrm{~S}$ nuclei, along the forward as well as along the backward direction. The process known as line scanning was performed with Leitz microscopes under a total magnification of 300x. On the other hand, Koristka microscopes were utilized for the track counting and angle measurement purposes, for which a total magnification of $1500 \mathrm{x}$ was used. The secondary charged particles coming out of an event are categorized in the following way.

(i) The shower tracks-caused by the singly charged produced particles most of which are $\pi$ mesons. In an event, their number is denoted by $n_{s}$.

(ii) The grey and black tracks-resulting from the fragments of the target $(\mathrm{Ag} / \mathrm{Br})$ nuclei. Their numbers are denoted, respectively, by $n_{g}$ and $n_{b}$, and the total number $n_{h}\left(=n_{g}+n_{b}\right)$ denotes the number of target fragments in an event.

(iii) The projectile fragments-caused by the spectator parts of the incident projectile $(\mathrm{Si} / \mathrm{S})$ nuclei. In an event, their number is denoted by $n_{\mathrm{pf}}$.

The details of emulsion experiments, track selection criteria, and data acquisition techniques are nicely elaborated in [27, 28]. To ensure that an interaction involves either an $\mathrm{Ag}$ or a $\mathrm{Br}$ nucleus as the target, in each event we impose a cut $n_{h}>8$. Thus, altogether $331{ }^{28} \mathrm{Si}-\mathrm{Ag} / \mathrm{Br}$ events and 200 ${ }^{32} \mathrm{~S}-\mathrm{Ag} / \mathrm{Br}$ events are selected for further analysis, which is confined only to the angular distribution of the shower tracks. The average shower track multiplicity $\left\langle n_{s}\right\rangle=52.67 \pm 1.33$ for the ${ }^{28}$ Si-sample, and $\left\langle n_{s}\right\rangle=217.19 \pm 6.16$ for the ${ }^{32} \mathrm{~S}$ sample. The pseudorapidity $(\eta)$ variable is an approximation of the dimensionless boost parameter rapidity, and it is related to the emission angle $(\theta)$ of a track as $\eta=-\ln \tan (\theta / 2)$. An accuracy of $\delta \eta=0.1$ unit is achieved through the reference primary method of angle measurement. For each data set, the $\eta$ distribution can be crudely approximated by a Gaussian function, whereas the azimuthal angle $(\varphi)$ distributions are in both cases more or less uniform between 0 and $2 \pi$. The Gaussian fit parameters for the $\eta$-distribution in the ${ }^{28} \mathrm{Si}$-sample are the peak density $\rho_{0}=17.88$, the centroid $\eta_{0}=1.90$, and the width $\sigma_{\eta}=2.17$. For the ${ }^{32} \mathrm{~S}$-sample they are $\rho_{0}=56.34, \eta_{0}=3.37$, and $\sigma_{\eta}=$ 1.55. Due to event averaging, the statistical noise as well as the nonstatistical components of the fluctuations present in individual events are simultaneously smoothed out in the overall distributions. Our basic task is, therefore, (i) to look for statistically significant unusual local structures in the particle distributions in individual $A B$ events and (ii) to study systematic collective behaviour in large samples of $A B$ events, if there is any.

\section{Simulation}

To eliminate the background noise, we compare the experiment with the UrQMD (version 3.3p1) model [22, 23]. UrQMD itself does not incorporate any kind of particle correlation, and therefore, in this regard it can be utilized only to generate the statistical background. The rationale behind using a transport model like the UrQMD is that it treats the final freeze-out stage dynamically. It does not make any equilibrium assumption and describes the dynamics of a hadron gas system very well in and out of the chemical 


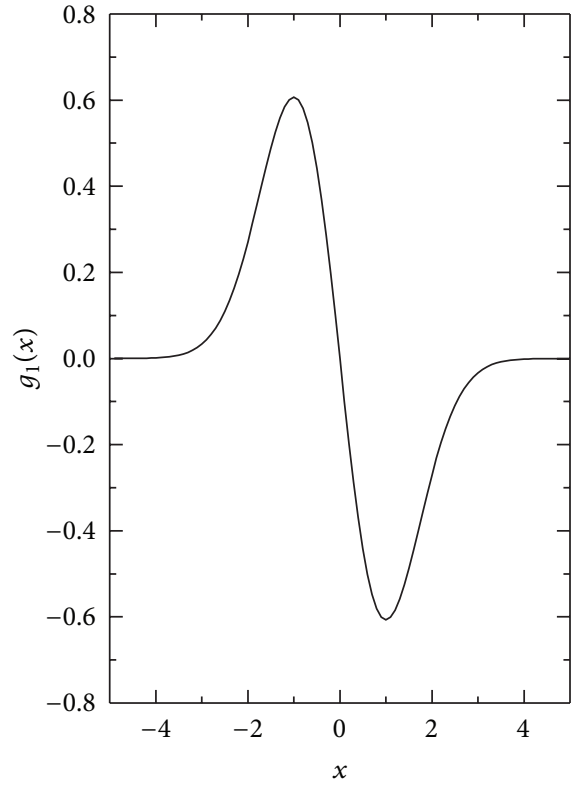

(a)

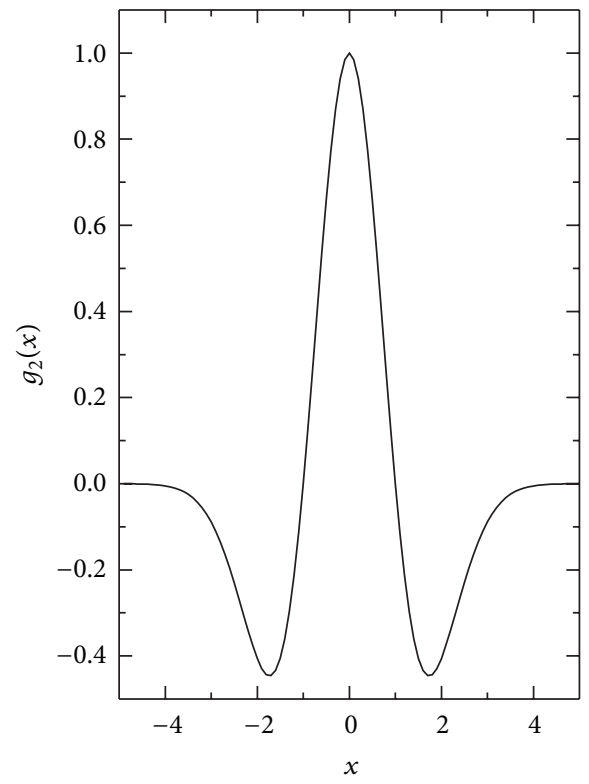

(b)

FIgURE 1: (a) First derivative and (b) second derivative (Mexican hat wavelet) of Gaussian function.

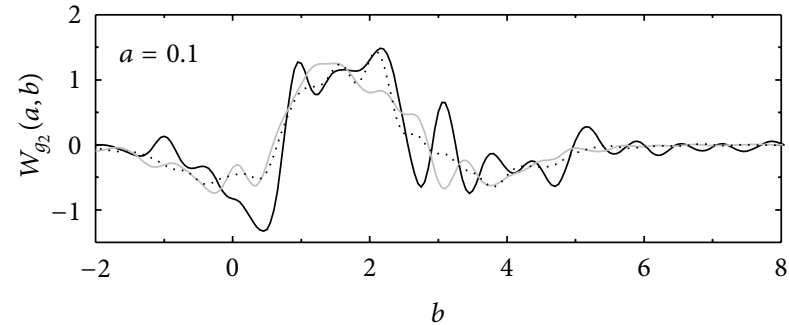

(a)

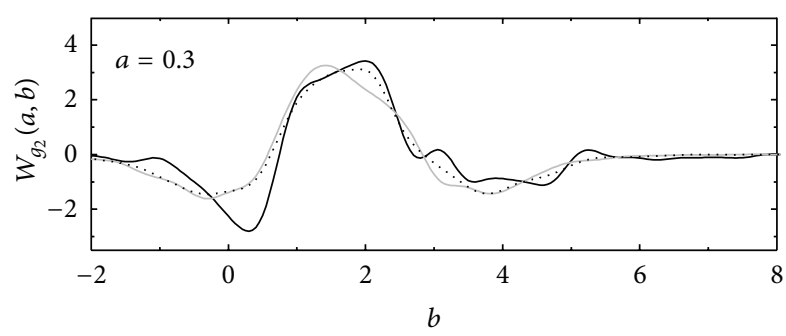

Experiment
UrQMD

UrQMD + BEC

(c)

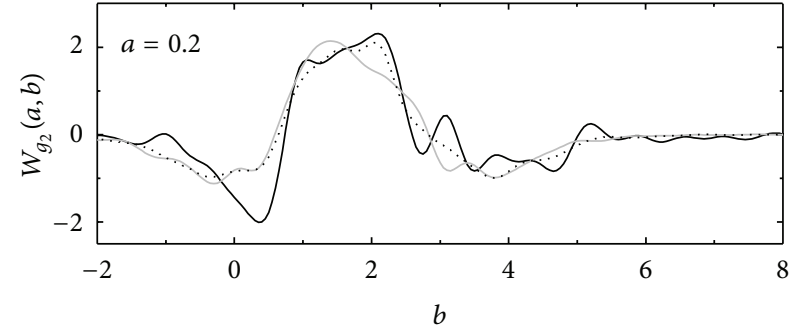

(b)

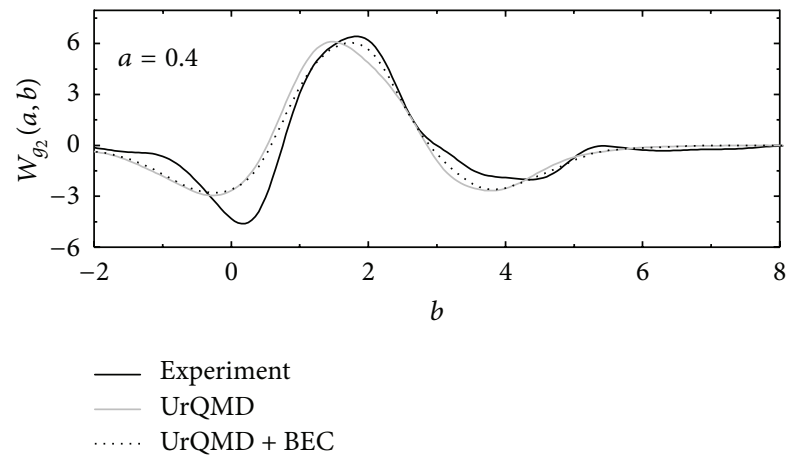

(d)

FIGURE 2: $g_{2}$ wavelet pseudorapidity spectra of ${ }^{28} \mathrm{Si}-\mathrm{Ag} / \mathrm{Br}$ interaction at $14.5 \mathrm{~A} \mathrm{GeV}$ for different values of the scale parameter $a$.

and/or thermal equilibrium. In the present case, neither the incident nuclei are too large nor the collision energies are extremely high. Hence, one can not be sure whether local thermal and/or chemical equilibrium are/is achieved. To describe such nonequilibrium many-body dynamics, a transport model is a natural choice. The UrQMD model is applicable over a wide range of energies starting from
$\sqrt{s_{N N}} \sim 5 \mathrm{GeV}$ and ending up at $\sqrt{s_{N N}}>200 \mathrm{GeV}$. In this scheme, particle production at high-energy interactions is implemented by the color string fragmentation mechanism similar to that of the Lund model. The UrQMD code has been successfully used to reproduce the particle density distributions and the transverse momentum $\left(p_{T}\right)$ spectra of various particle species in proton-proton, proton-nucleus, 


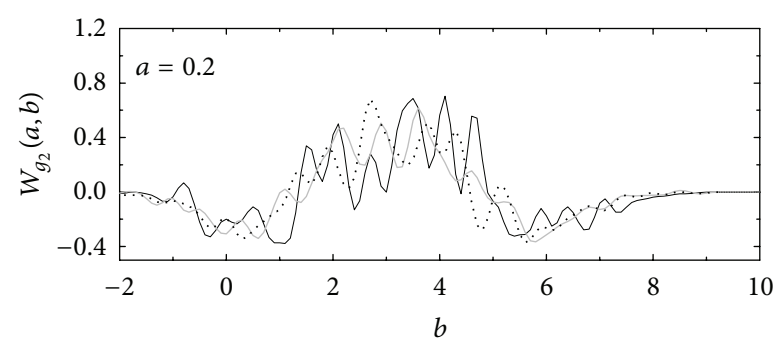

(a)

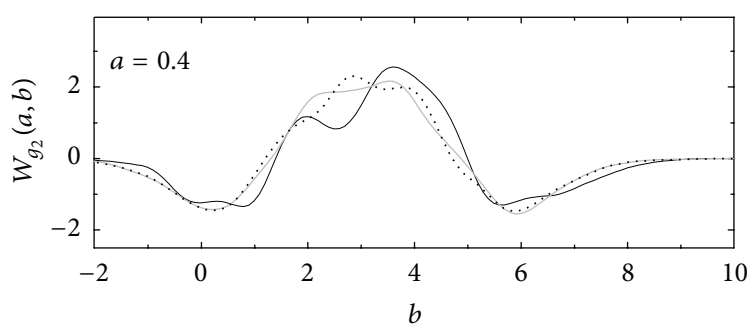

$\begin{array}{ll} & \text { Experiment } \\ & \text { UrQMD } \\ \ldots . . & \text { UrQMD + BEC }\end{array}$

(c)

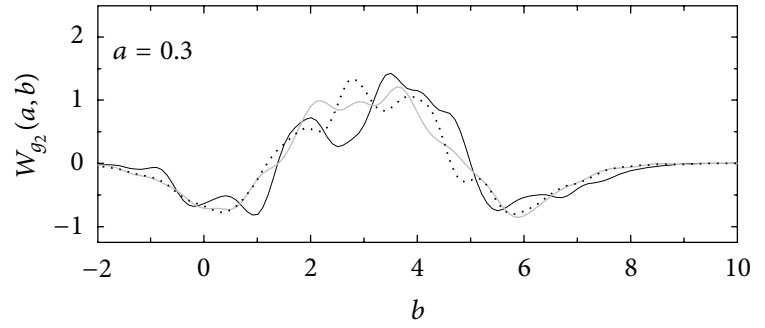

(b)

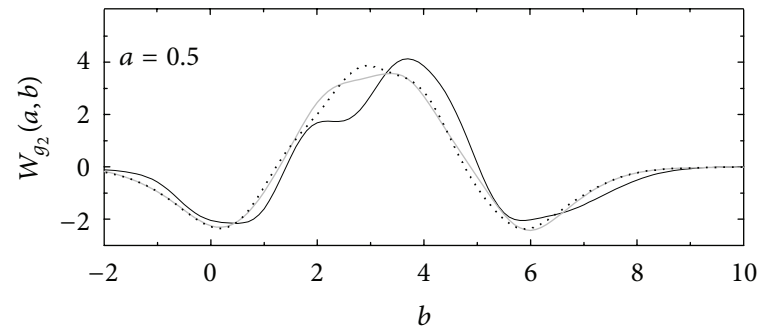

$\begin{array}{ll}- & \text { Experiment } \\ \text { UrQMD } \\ \text { …. } & \text { UrQMD + BEC }\end{array}$

(d)

FIGURE 3: $g_{2}$ wavelet pseudorapidity spectra of ${ }^{32} \mathrm{~S}-\mathrm{Ag} / \mathrm{Br}$ interaction at $200 \mathrm{~A} \mathrm{GeV}$ for different values of the scale parameter $a$.

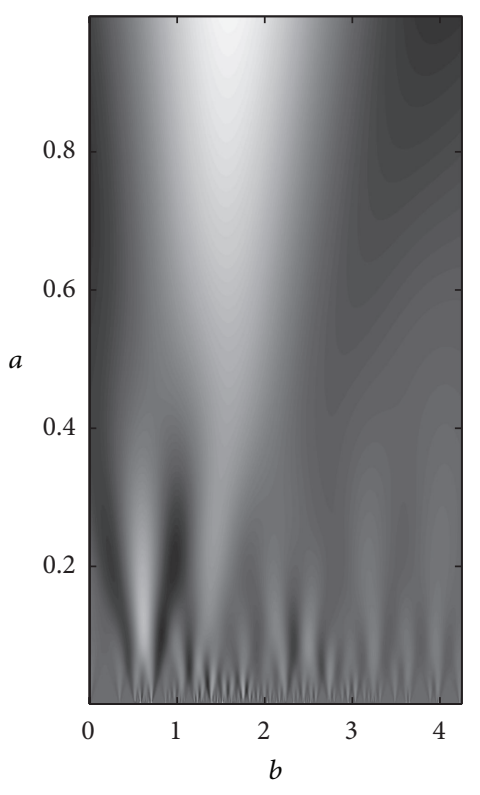

(a)

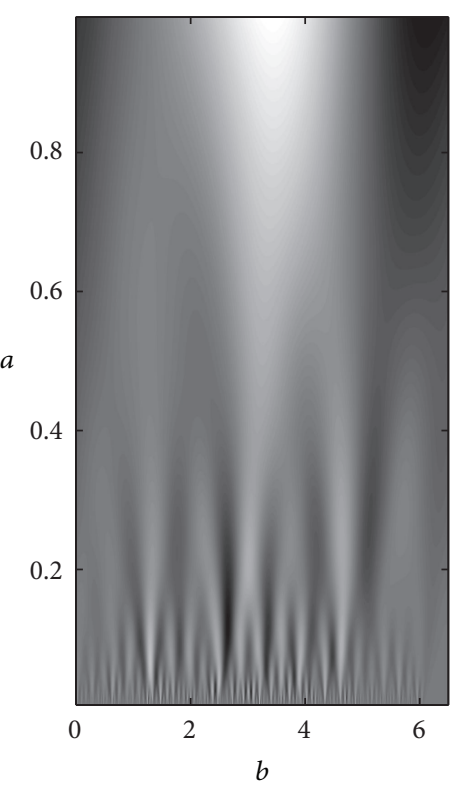

(b)

FIGURE 4: Wavelet pseudorapidity spectra for a single event (a) in ${ }^{28} \mathrm{Si}-\mathrm{Ag} / \mathrm{Br}$ interaction at $14.5 \mathrm{~A} \mathrm{GeV}$ : event multiplicity 146 , and (b) in ${ }^{32} \mathrm{~S}-\mathrm{Ag} / \mathrm{Br}$ interaction at $200 \mathrm{~A} \mathrm{GeV}$ : event multiplicity 379.

and $A B$ collisions. However, as mentioned above, the model does not incorporate the symmetry aspects of the fields associated with the produced particles.

It is well known that the Bose-Einstein correlation (BEC), an identical particle effect, dominates the origin of cluster formation. Due to the correlated emission of like sign and/or opposite sign mesons, the particle yield with small relative momenta may be enhanced, which is one of the reasons of large local densities in the final state particles in any high-energy interaction. The effect is quantum statistical in nature and it is not incorporated in the framework of a transport model like the UrQMD. Recently, a new algorithm 


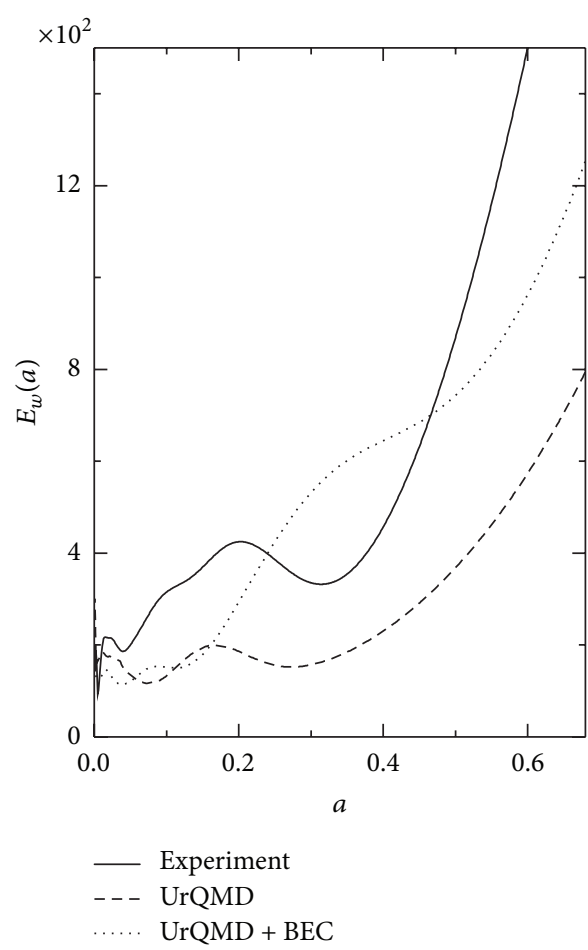

(a)

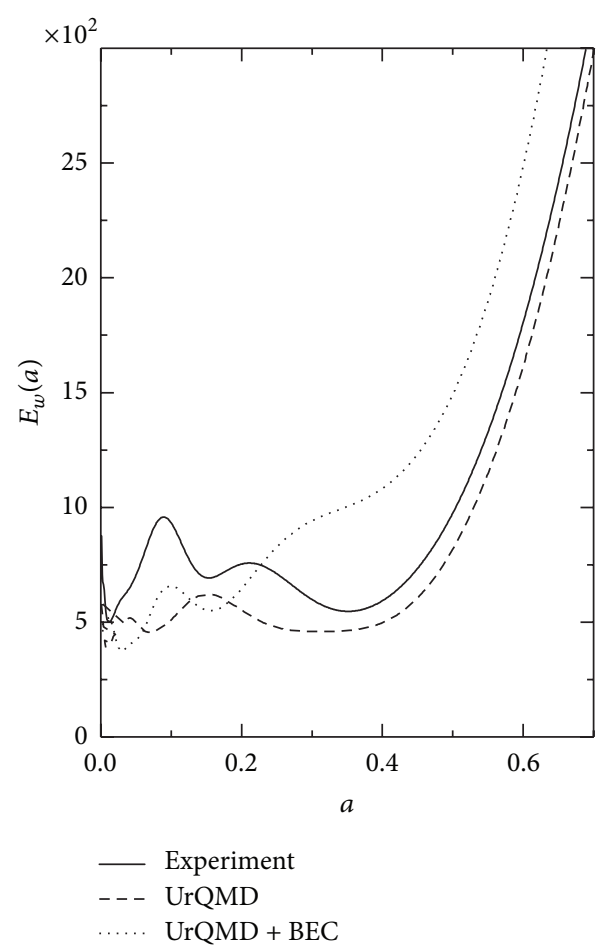

(b)

FIGURE 5: Scalogram for a single event: (a) ${ }^{28} \mathrm{Si}-\mathrm{Ag} / \mathrm{Br}$ interaction at $14.5 \mathrm{~A} \mathrm{GeV}$ : event multiplicity 146 , and (b) ${ }^{32} \mathrm{~S}-\mathrm{Ag} / \mathrm{Br}$ interaction at $200 \mathrm{~A} \mathrm{GeV}$ : event multiplicity 379 . The same events for which the wavelet pseudorapidity spectra are shown in Figure 4 are used for drawing the scalograms.

has been developed $[24,25]$, where the BEC is introduced by reassigning the charges of produced mesons in such a way that the overall phase-space distribution in each simulated event remains unaltered. The event-wise particle multiplicities are not changed, and it looks as if the particles (mesons) are satisfying the BE statistics. The method of numerically modeling the BEC at the level of a so-called "after burner," where the output of the UrQMD code is used, is very briefly described below. The UrQMD code provides the four coordinates and the four momenta of all particles. The particle information are contained in an ASCII file written in the OSCAR format. Each particle entry in an event contains a serial number, a particle ID, the particle four momentum $\left(p_{x}, p_{y}, p_{z}, E\right)$, the particle mass $m$, and the final freeze-out four coordinates $(x, y, z, t)$.

(i) In the first step, we arbitrarily choose a meson from an event, and irrespective of its original charge, assign a charge "sign" $c=+,-$ or 0 to it with weight factor $p_{c}=$ $n_{c} / n$. Here, $n_{+}, n_{-}, n_{0}$ are the numbers, respectively, of the $+\mathrm{ve},-\mathrm{ve}$ and neutral mesons, and $n\left(=n_{+}+n_{-}+n_{0}\right)$ is the total number of mesons in the event. The chosen meson, say the $i$ th one, defines a phase-space cell.

(ii) In the next step, we calculate the distances in the four momenta $\delta_{i j}(p)=\left|p_{i}-p_{j}\right|$ and the four coordinates $\delta_{i j}(x)=\left|x_{i}-x_{j}\right|$ between the already chosen meson (i.e., the $i$ th one) and all other mesons (indexed by $j$ ) that are not yet assigned any charge "sign." Each $j$ th meson is associated with a weight factor [24]

$$
P_{i j}=\exp \left[-\frac{1}{2} \delta_{i j}^{2}(x) \delta_{i j}^{2}(p)\right],
$$

which characterizes the bunching probability of the particles in a given cell.

(iii) Then, we start to generate uniformly distributed random numbers $r \in(0,1)$. If $r<P_{i j}$, we reassign the same charge "sign" to the jth meson and put it in the same phase-space cell as the $i$ th one. We continue the process until either (a) $r$ exceeds $P_{i j}$ or (b) all mesons in the event having same charge "sign" as the $i$ th one are exhausted.

(iv) Now, we go back to our first step and again randomly choose a meson from the pool of the left over mesons for which the charge reassignment has not yet been done. Obviously, the weight factors $p_{ \pm, 0}$ will now be updated, as some of the particles present in the event are already used up.

(v) The algorithm is then repeated until all mesons belonging to each charge variety in the event are used up, and then we move to the next event.

Only the meson pairs with space-like separation are accepted, and appropriate checks are imposed so that $P_{i j}$ does not 


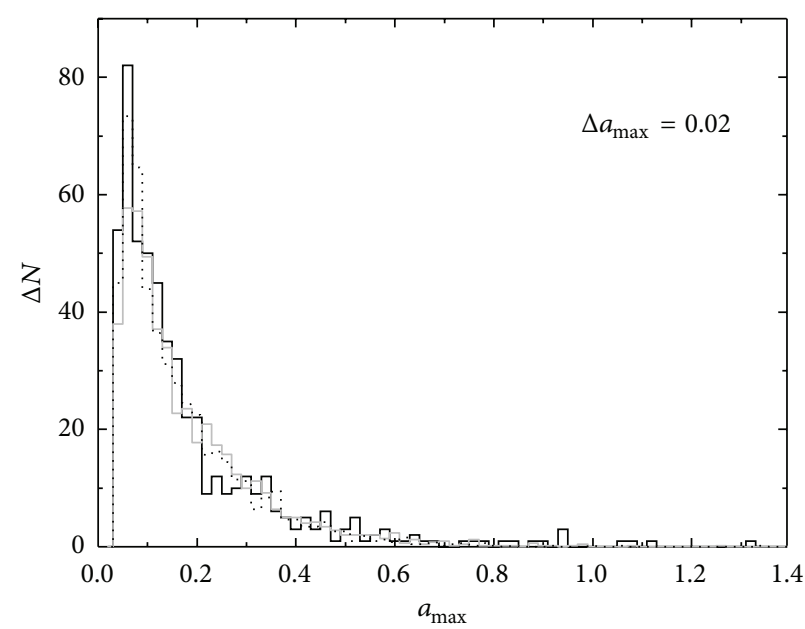

(a)

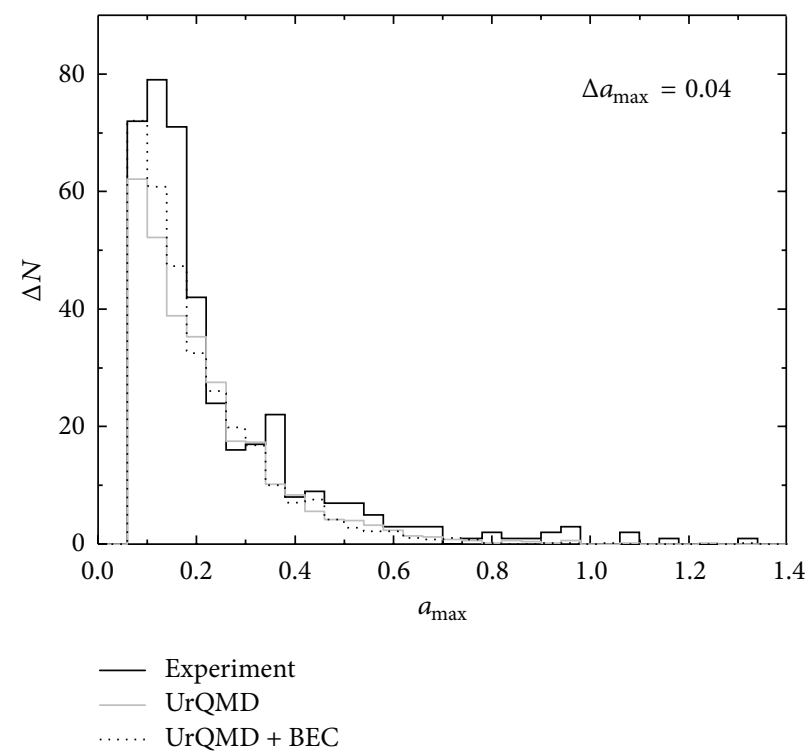

(b)

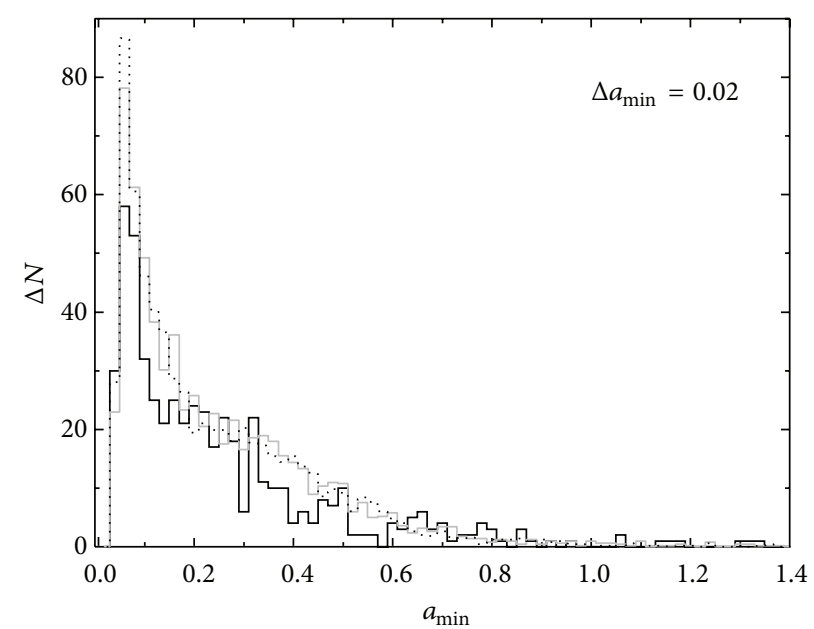

(c)

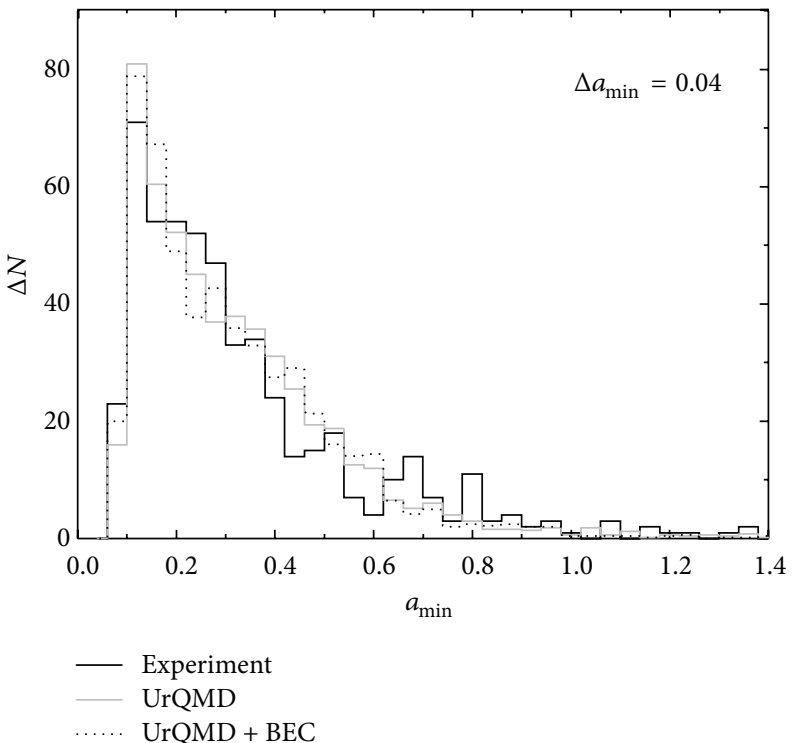

(d)

FIGURE 6: Distributions of the local maxima (left panel) and minima (right panel) of the scalograms for ${ }^{28} \mathrm{Si}-\mathrm{Ag} / \mathrm{Br}$ interaction at $14.5 \mathrm{~A}$ GeV.

exceed unity [26]. Without changing the overall set of four momenta, four coordinates, or total mesonic charge of the system, we can in this way generate clusters of closely spaced identical charge states of mesons.

We use the UrQMD code in its default setting and generate the minimum bias event samples in the laboratory frame, separately for the $\mathrm{Ag}$ and the $\mathrm{Br}$ targets and, respectively, for the ${ }^{28} \mathrm{Si}$ and ${ }^{32} \mathrm{~S}$ projectiles. For each projectile, the Ag and $\mathrm{Br}$ event samples are then mixed up. While doing so, the proportional abundances of these nuclei in the G5 emulsion [28] are maintained. Only the produced charged mesons are retained for subsequent analysis. From the minimum bias samples, we select subsamples in such a way as to match the respective experimental $n_{s}$-distributions. For each projectile, the final sample of simulated events is five times as large as the corresponding experimental one, and the corresponding normalized $\eta$ and/or $\varphi$ distributions can be approximately described by more or less the same set of parameters as quoted above for the respective experiment. The UrQMD events are then passed through the charge reassignment algorithm as mentioned above, and from now on these modified samples will be known as the UrQMD + BEC samples.

\section{Wavelet Analysis}

The wavelet method is used to analyze nonstationary as well as inhomogeneous signals that can be any ordered set of numerically recorded information on some processes, objects, functions, and so forth. A wavelet construction is based on a dilation ( $a$ ) and a translation ( $b$ ) parameter. By changing $a$, the local characteristics of a signal are distinguished, while by doing the same with $b$ the whole range of a spectrum is analyzed. Unlike the Fourier transformation 


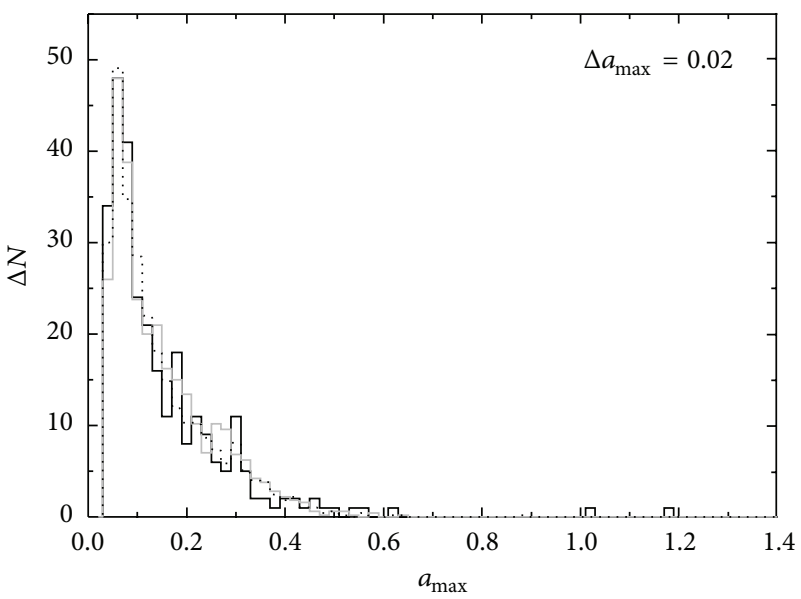

(a)

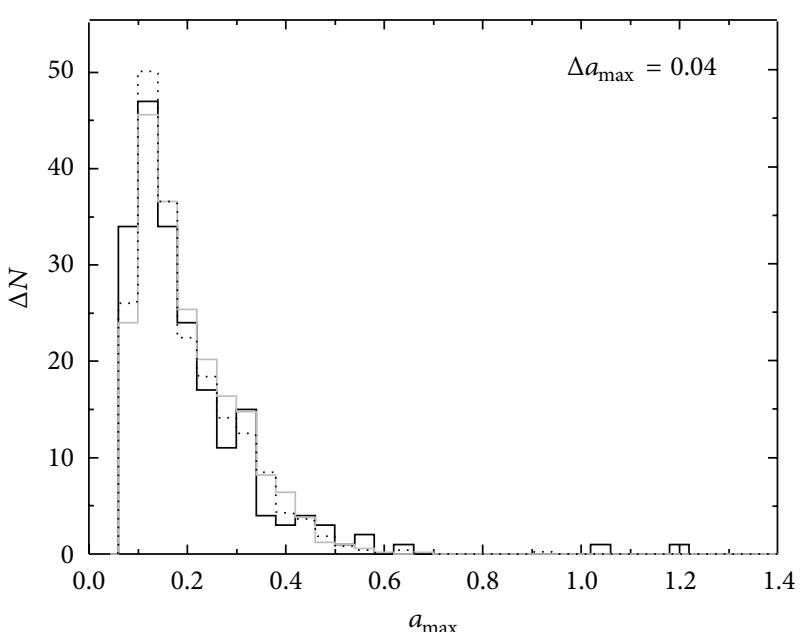

Experiment

UrQMD

$\mathrm{UrQMD}+\mathrm{BEC}$

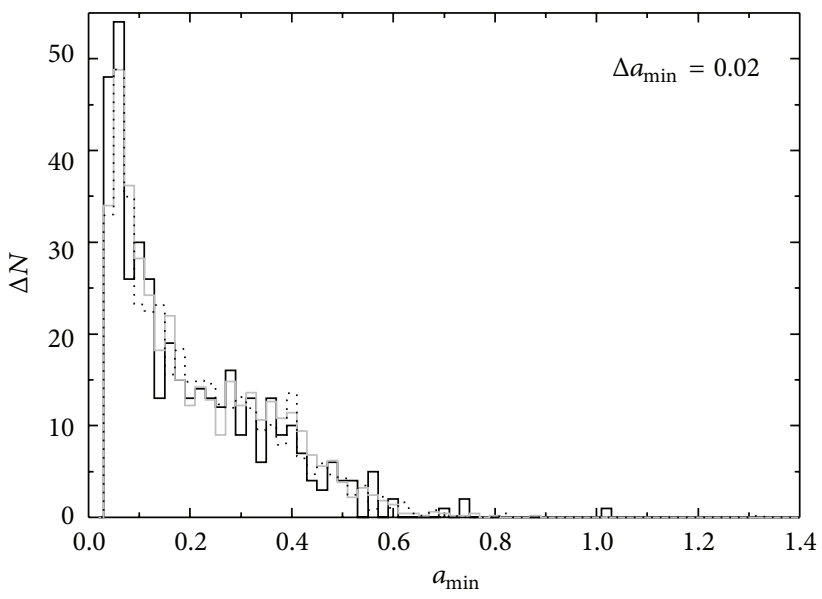

(c)

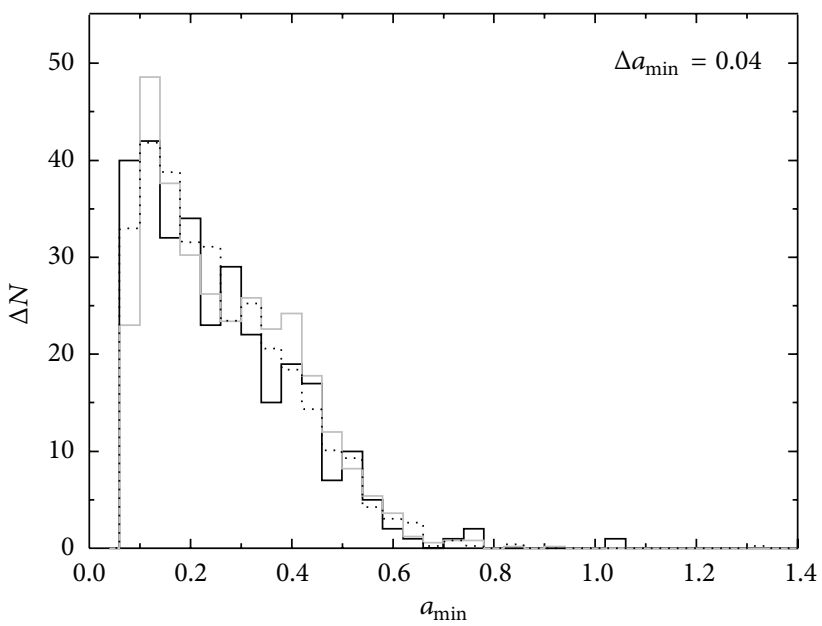

Experiment
UrQMD
UrQMD + BEC

(b)

(d)

Figure 7: Same as Figure 6 but for ${ }^{32} \mathrm{~S}-\mathrm{Ag} / \mathrm{Br}$ interaction at $200 \mathrm{~A} \mathrm{GeV}$.

method which uses only two basis functions, the wavelet transformation method can in principle use an infinite set of discrete or continuous functions as the basis. However, a suitable choice of the basic wavelet is made only after looking at the basic features of the signal to be processed. In the present case, we use a continuous wavelet to find out the strongest fluctuations on an event by event basis that may exceed the expected statistical noise at a particular scale and at a particular point of the underlying phase-space variable, say $x$. The wavelet transform of a function $f(x)$ is its decomposition into an orthogonal functional family $(\Psi)$ like

$$
W_{\Psi}(a, b) f=\frac{1}{\sqrt{C_{\Psi}}} \int_{-\infty}^{+\infty} f(x) \Psi_{a, b}(x) d x,
$$

where $\Psi_{a, b} \equiv(1 / \sqrt{a}) \Psi((x-b) / a)$ is the wavelet characterized by $a$ and $b$ as mentioned above, $C_{\Psi}=2 \pi \int_{-\infty}^{+\infty}|\widetilde{\Psi}(\omega)|^{2}|\omega|^{-1} d \omega$ is a normalisation constant, and $\widetilde{\Psi}(\omega)$ is the Fourier transform of $\Psi(x)$. Derivatives of the Gaussian function

$$
\Psi(x) \equiv g_{n}(x)=(-1)^{n+1} \frac{d^{n}}{d x^{n}} e^{-x^{2} / 2}
$$

are often used as mother wavelets. In particular, the second derivative:

$$
g_{2}(x)=\left(1-x^{2}\right) e^{-x^{2} / 2},
$$

popularly known as the Mexican hat (MHAT) distribution, is customarily used to analyze multiparticle emission data. In Figure 1, we show the plots of $g_{1}(x)$ and $g_{2}(x)$. In the present case, the phase-space variable is $\eta$ and the signal to be analyzed is the density function

$$
f(\eta)=\frac{d n}{d \eta}=\sum_{i=1}^{N} \delta\left(\eta-\eta_{i}\right)
$$




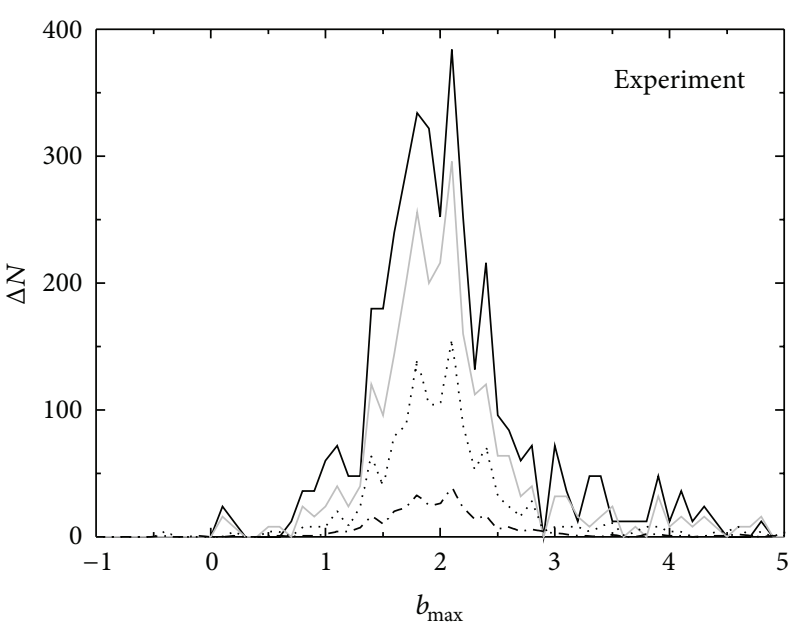

(a)

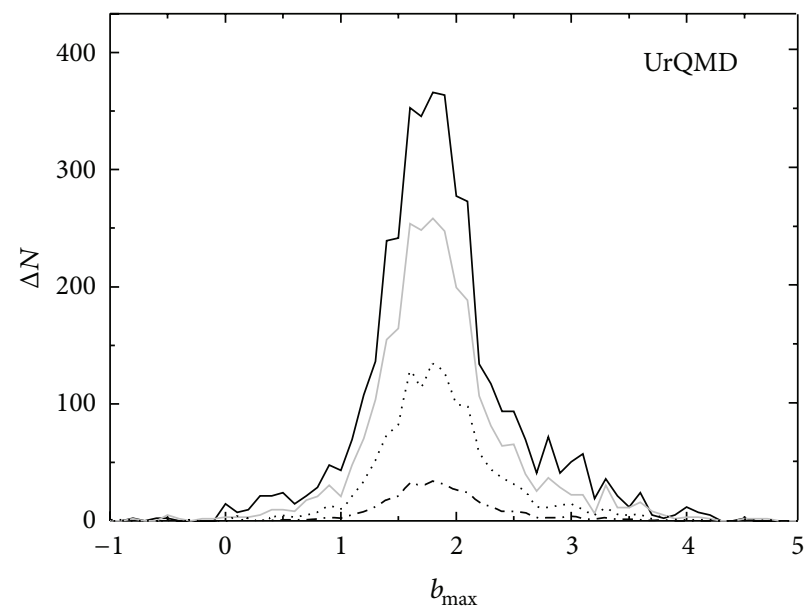

(b)

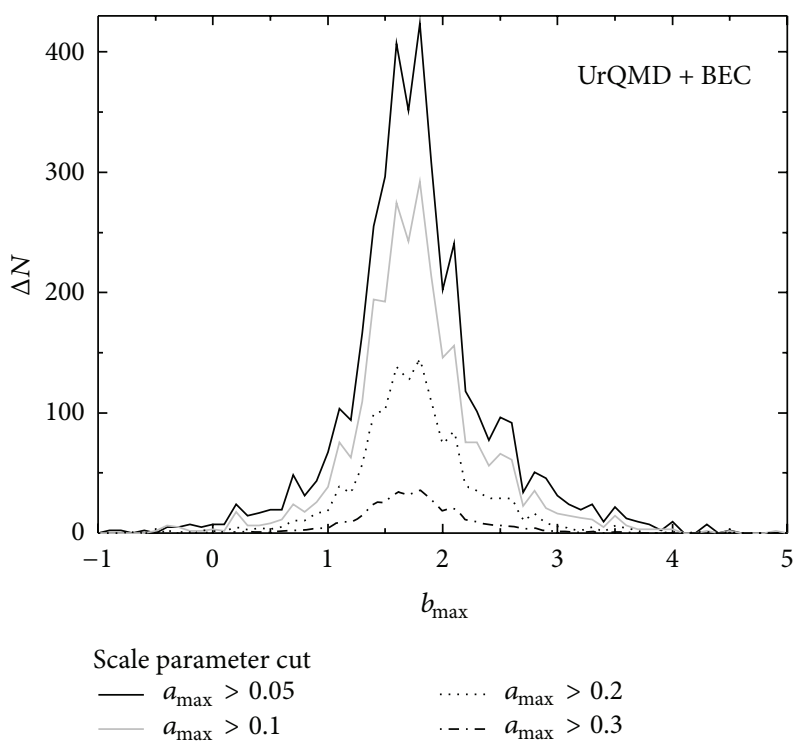

(c)

FIGURE 8: $b_{\max }$ distributions for different scale windows in ${ }^{28} \mathrm{Si}-\mathrm{Ag} / \mathrm{Br}$ interaction at $14.5 \mathrm{~A} \mathrm{GeV}-$ (a) the experiment, (b) the UrQMD, and (c) the UrQMD + BEC. The distributions in different scale windows are so shifted as to avoid mutual overlapping.

where $N$ is the number of shower tracks in the event sample considered and $\eta_{i}$ is the pseudorapidity of the $i$ th particle. $N$ may either be the $n_{s}$ value of a single event, or it may be the total number of shower tracks present in the entire event sample/subsample within the $\eta$ interval considered. The wavelet transform of $f(\eta)$, therefore, becomes

$$
W_{\Psi}(a, b) f=\frac{1}{N} \sum_{i=1}^{N} \frac{1}{\sqrt{a}} \Psi\left(\frac{\eta_{i}-b}{a}\right) .
$$

$W_{\Psi}(a, b)$ is the contribution of $\Psi(a, b)$ to the spectrum $f(\eta)$ in the sense that it represents the probability to find out a particle at some $b=\eta_{i}$ at the scale $a$. A wavelet image at large scale shows only the coarse features, while the same at small $a$ reveals the more detailed and finer structures of the underlying distribution.

\section{Results}

In Figure 2, we have presented the $g_{2}$ pseudorapidity spectra of the shower tracks coming out of all $331{ }^{28} \mathrm{Si}-\mathrm{Ag} / \mathrm{Br}$ events at an incident energy of $14.5 \mathrm{~A} \mathrm{GeV}$ at different scales (four different $a$ values). For comparison, the UrQMD and the UrQMD + BEC graphs are plotted along with those of the experiment. Though the overall multiplicity and the $\eta$ distributions of the simulated and experimental event samples are identical, we observe that the $g_{2}$ spectra of the experiment are quite different from those of the simulations. The fluctuations are more rapid in the experiment. In Figure 2(a), we can see peaks at $b \approx 1.0,2.0$ and 3.0 in the experimental distribution. These are the preferred $\eta$ values where particle clusters are formed, and one can relate them, respectively, to the target fragmentation, the central particle producing, and 


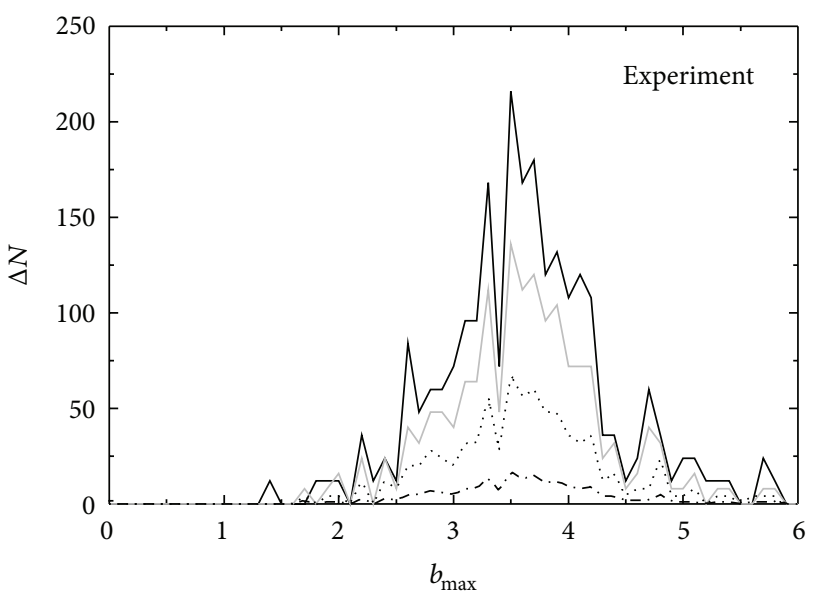

(a)

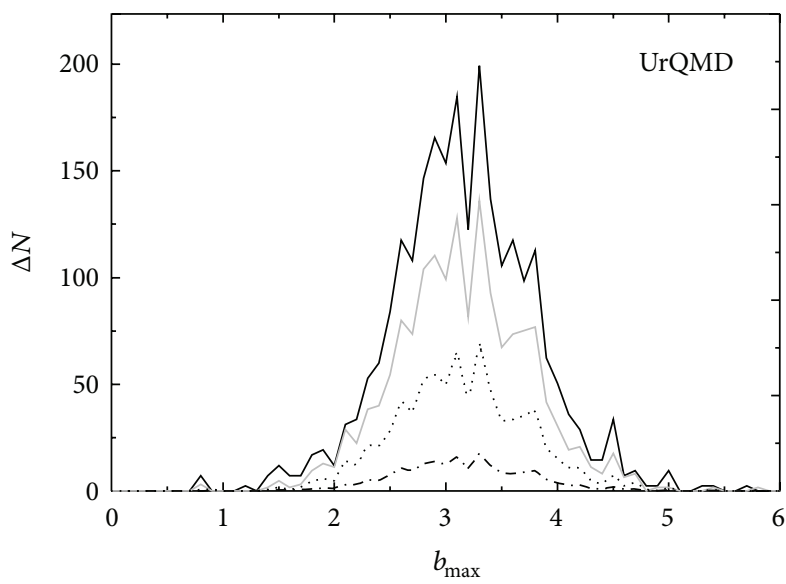

(b)

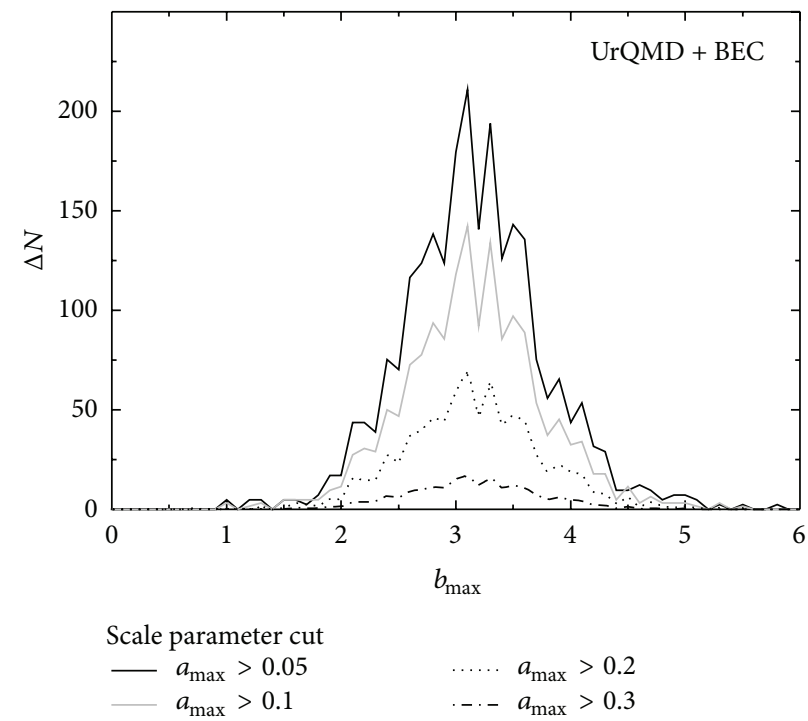

(c)

Figure 9: The same as Figure 8 but for ${ }^{32} \mathrm{~S}-\mathrm{Ag} / \mathrm{Br}$ interaction at $200 \mathrm{~A} \mathrm{GeV}$.

the projectile fragmentation regions. However, we also notice that the central particle producing peak around $b \approx 2.0$ is well reproduced by the UrQMD + BEC plot. As expected, with increasing $a$ the fluctuations are smoothed out, and the distributions gradually converge to the mother wavelet $g_{2}$. Needless to say that such plots do not reflect any unique structure of particle distribution in individual events. They would rather correspond to a systematic collective behaviour of the particle emission of the entire sample, if there is any. Similar plots for all the entire ${ }^{32} \mathrm{~S}-\mathrm{Ag} / \mathrm{Br}$ event sample at $200 \mathrm{~A} \mathrm{GeV} / \mathrm{c}$ are presented in Figure 3. While the general features of Figures 2 and 3 are more or less similar, we notice that more peaks are present in the ${ }^{32} \mathrm{~S}$-sample than in the ${ }^{28} \mathrm{Si}$-sample. There are at least 6 prominent peaks within $b \approx$ 1.0-5.0 in the experiment, out of which two very prominent peaks are lying within the central particle producing region $(b \approx 3.0-4.0)$, and the simulations can not replicate them. Even at a large scale $a(=0.5)$, we find a hump to the left of the peak of the experimental distribution that refuses to be smoothed out, a feature that is absent in the ${ }^{28} \mathrm{Si}$ case. The other peaks, one to the right and three to the left side of the central region, can be related, respectively, to the projectile and the target fragmentations.

The wavelet spectra can be generated for individual events at many different scales that can be used to simultaneously study the location and scale dependence of $W_{\Psi}(a, b)$. We have obtained such distributions for two high multiplicity events, one for a ${ }^{28} \mathrm{Si}-\mathrm{Ag} / \mathrm{Br}$ event $\left(n_{s}=146\right)$ and the other for a ${ }^{32} \mathrm{~S}-\mathrm{Ag} / \mathrm{Br}$ one $\left(n_{s}=379\right)$. We have schematically presented the $W_{\Psi}(a, b)$ distributions, respectively, in Figures 4(a) and 4(b). The dark (white) regions in the graphs correspond to the low (high) $W_{\Psi}(a, b)$ values. As mentioned before, at the finest scales $(a<0.05)$ we only get information about individual particles while at large $a$ particles loose their individual identities to coalesce into a big cluster. It is, therefore, pointless to study any event at these two extreme 


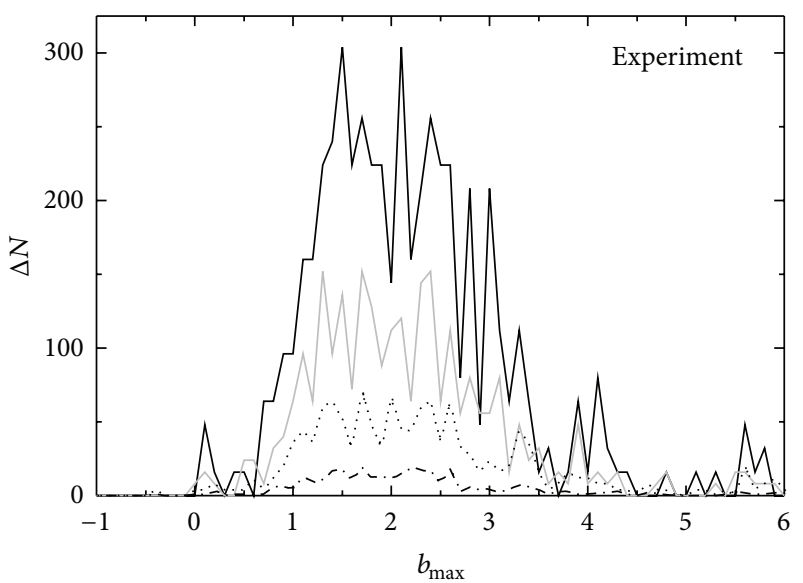

(a)

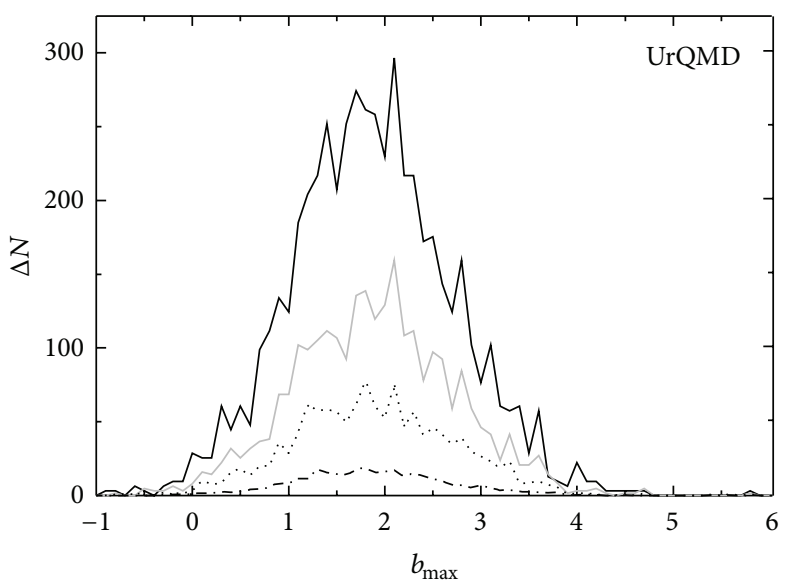

(b)
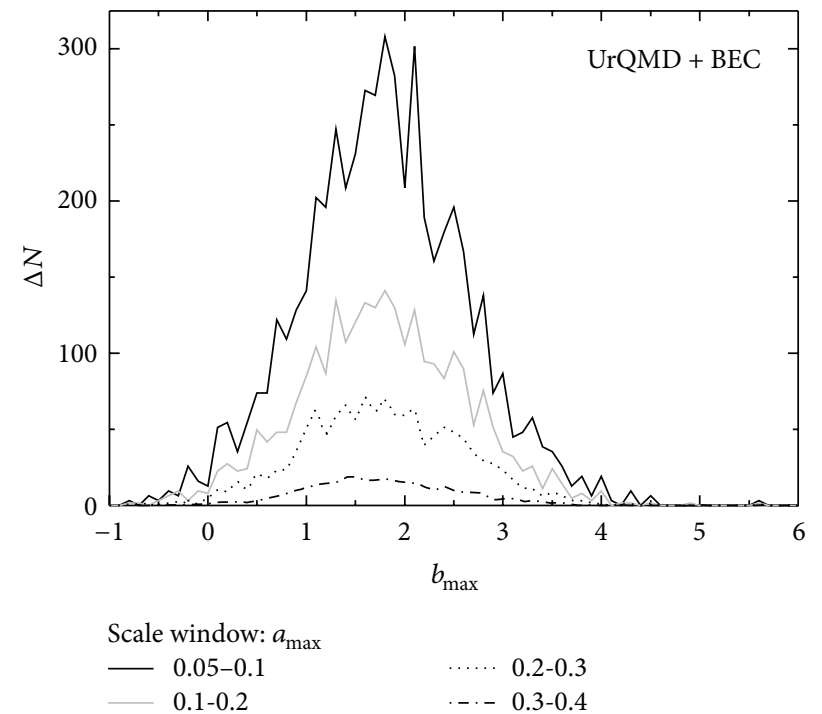

(c)

FIGURE 10: $b_{\max }$ distributions for different scale windows in ${ }^{28} \mathrm{Si}-\mathrm{Ag} / \mathrm{Br}$ interaction at $14.5 \mathrm{~A} \mathrm{GeV}-(\mathrm{a})$ the experiment, (b) the UrQMD, and (c) the UrQMD + BEC. The distributions for different scale windows are so shifted as to avoid mutual overlapping.

but trivial scales. We see that in both diagrams several small and large clusters are present at $a>0.1$. Looking at the ${ }^{28} \mathrm{Si}-\mathrm{Ag} / \mathrm{Br}$ diagram, we recognize that two large groups of particles are present, one centered around $\eta \approx 0.6$ and the other around $\eta \approx 1.4$. Similarly in the ${ }^{32} \mathrm{~S}-\mathrm{Ag} / \mathrm{Br}$ diagram again, there are two large groups, one at $\eta \approx 3.0$ and the other at $\eta \approx 4.6$. Beside them several other smaller groups of particles are present, all belonging to the fragmentation regions.

Identification of the peculiarities in particle distribution in individual events from the 2-d energy spectrum $\left\{W_{\Psi}(a, b)\right\}^{2}$ is a difficult proposition. Instead we may concentrate on the scalogram $E_{w}(a)$ defined as

$$
E_{w}(a)=\int\left\{W_{\Psi}(a, b)\right\}^{2} d b,
$$

which represents the 1-d energy distribution with respect to the scale (a). A scalogram reflects some of the characteristic features of an event. As, for example, a minimum on it represents the average distance between the particle clusters, while a maximum represents the most compact groups of particles present. Two such scalograms, one for the ${ }^{28} \mathrm{Si}-\mathrm{Ag} / \mathrm{Br}$ event and the other for the ${ }^{32} \mathrm{~S}-\mathrm{Ag} / \mathrm{Br}$ event considered above, are plotted in Figure 5. In each diagram, a peak or a small rise seen at the lowest scale represents individual particles, and they are of little significance. In the scalogram of the ${ }^{28} \mathrm{Si}$ $\mathrm{Ag} / \mathrm{Br}$ event a peak at $a \approx 0.2$ and a minimum at $a \approx 0.3$ are seen. On the other hand, in the ${ }^{32} \mathrm{~S}-\mathrm{Ag} / \mathrm{Br}$ event there are a couple of maxima and minima. The maxima are located at $a \approx 0.1$ and 0.2 , while the minima are located at $a \approx$ 0.15 and 0.35 . The simulation, either with or without BEC, fails to reproduce the experiment at the significant scales. It is now amply clear that the scales and the $\eta$ values at which the clusters are formed will vary from one event to the other. Most of the local maxima (minima) are found within $a \approx 0.1-0.5$, and in most of the events only a few 


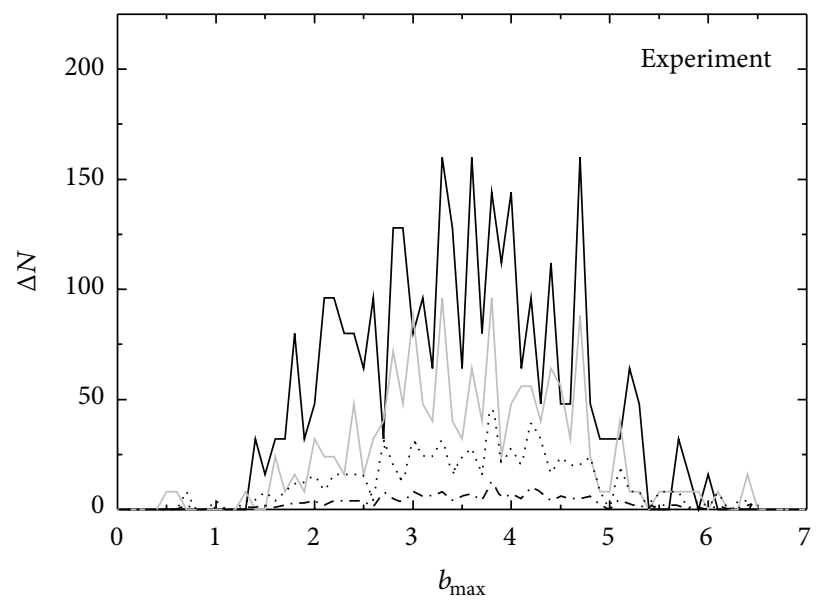

(a)

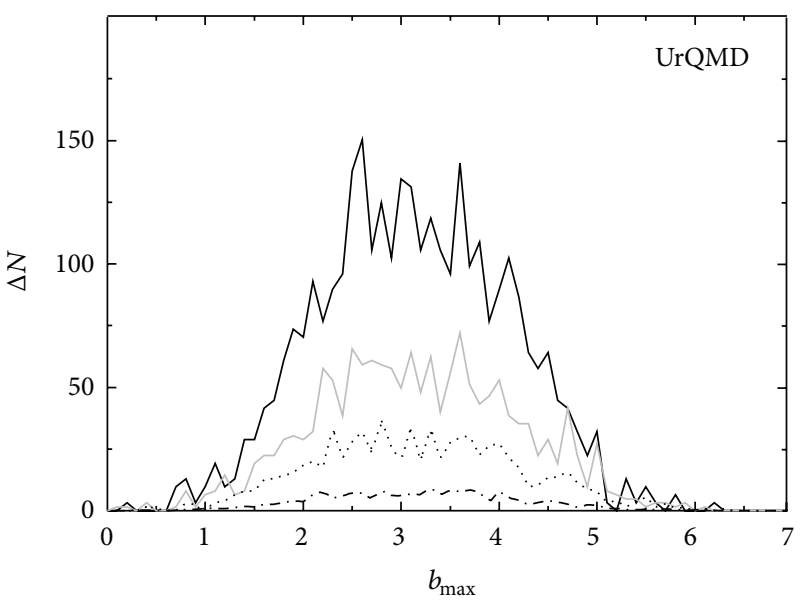

(b)

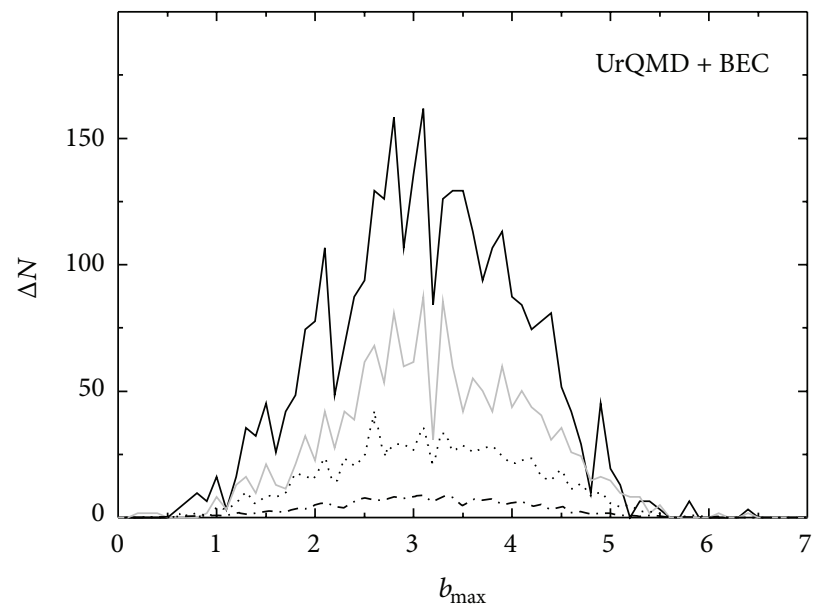

Scale window: $a_{\max }$

(c)

FIgUre 11: The same as Figure 10 but for ${ }^{32} \mathrm{~S}-\mathrm{Ag} / \mathrm{Br}$ interaction at $200 \mathrm{~A} \mathrm{GeV}$.

such maxima (minima) are found. To check whether there exists any systematic behaviour of particle emission, or the clusters occur at random, we investigate the distributions of the extremum points over our entire event sample(s). In Figure 6, we plot the frequency distribution of the scales $a_{\max }$ and $a_{\min }$ at which, respectively, the maxima and the minima of the scalograms belonging to individual ${ }^{28} \mathrm{Si}-\mathrm{Ag} / \mathrm{Br}$ events are graphically seen. The experiments as usual are plotted together with the simulations. Except in Figure 6(b), where the experiment slightly exceeds the simulation at the characteristic scale of $a_{\max } \approx 0.2$, no significant difference between experiment and simulation is observed. In Figure 7, similar histograms for the ${ }^{32} \mathrm{~S}-\mathrm{Ag} / \mathrm{Br}$ events are plotted. In this case also no significant difference between the experiment and the corresponding simulation is seen.

The wavelet analysis is not complete unless we study the distributions of the locations $(b)$, where the local maxima in $W_{\Psi}\left(a_{\max }, b_{\max }\right)$ are observed. We do this with different choices of scale intervals, cumulative as well as differential. In Figure 8, such distributions for the ${ }^{28} \mathrm{Si}-\mathrm{Ag} / \mathrm{Br}$ sample (both experiment and simulation) are graphically presented at different cumulative scale windows. The common features of these diagrams are that at the lowest $a_{\max }$ range the distributions are rapidly fluctuating, and as expected with increasing scale window size the fluctuations get reduced. In comparison with the experiment, the UrQMD distributions vary more smoothly. However, when the BEC is incorporated into the UrQMD, to some extent the fluctuating patterns are retrieved. The ${ }^{32} \mathrm{~S}-\mathrm{Ag} / \mathrm{Br}$ sample on the other hand behaves a little differently. The distributions are shown in Figure 9. In this case, the experiment is still more fluctuating than both the UrQMD and the UrQMD + BEC plots. However, incorporating BEC into UrQMD apparently has little effect on the respective distributions. In Figures 10 and 11, the $b_{\max }$ distributions, respectively, for the ${ }^{28} \mathrm{Si}-\mathrm{Ag} / \mathrm{Br}$ and ${ }^{32} \mathrm{~S}-$ $\mathrm{Ag} / \mathrm{Br}$ samples are once again graphically shown, where we 
choose differential scale intervals to draw the histograms. For both sets of data, the basic features are more or less the same. As expected at the smallest scale $0.05 \leq a_{\max } \leq 0.1$ most rapid fluctuations are seen, which are systematically smoothed out with increasing $a_{\max }$. The distributions for the ${ }^{32} \mathrm{~S}-\mathrm{Ag} / \mathrm{Br}$ interaction are slightly wider than those for the ${ }^{28} \mathrm{Si}-\mathrm{Ag} / \mathrm{Br}$ interaction. It seems that the inclusion of BEC into the UrQMD in both interactions increases the heights of the local peaks to a small extent.

\section{Conclusion}

Pseudorapidity distributions of singly charged particles coming out with relativistic speeds from the ${ }^{28} \mathrm{Si}-\mathrm{Ag} / \mathrm{Br}$ and ${ }^{32} \mathrm{~S}$ $\mathrm{Ag} / \mathrm{Br}$ interactions, respectively, at $14.5 \mathrm{~A} \mathrm{GeV}$ and $200 \mathrm{~A} \mathrm{GeV}$, are analyzed by using the continuous wavelet transform technique. Compared to similar other such emulsion investigations [17-21], the target nuclei in the present case have less uncertainties.

For background noise elimination, the experiments are compared with a set of ordinary UrQMD simulated data, and also with the same set of UrQMD output that is modified by a mimicry of the Bose-Einstein type of correlation. The observed discrepancies between the experiment and the corresponding simulation should, therefore, result from nontrivial dynamics like collective flow of hadronic matter.

Irregularities in the wavelet pseudorapidity spectra, not reproducible by the simulation, are observed in individual $A B$ events, and the cluster characteristics are not reproducible by the simulations. As far as systematic behavior in many events is concerned, we observed certain differences between experiment and simulation in the ${ }^{28} \mathrm{Si}$ event sample under consideration. The differences with all probability are not a result of ordinary correlations among identical bosons. They should be interpreted in terms of certain nontrivial dynamical reason(s), which are not very much clear from the present analysis.

The present study can be extended to the azimuthal angle distribution of the $\eta$ irregularities and to the $2-\mathrm{d}$ wavelet analysis with larger statistics, so that the impact parameter dependence of the observed irregularities can also be investigated.

\section{Acknowledgments}

Provash Mali acknowledges financial support from the University Grants Commission, Government of India; Project no. 40-510/2011 (SR). Soumya Sarkar is supported by the INSPIRE fellowship of the Department of Science \& Technology, Government of India.

\section{References}

[1] E. V. Shuryak, "Quantum chromodynamics and the theory of superdense matter," Physics Reports C, vol. 61, no. 2, pp. 71-158, 1980.

[2] K. Yagi, T. Hatsuda, and Y. Make, Quark-Gluon Plasma, Cambridge University Press, New York, NY, USA, 2005.
[3] Y.-G. Ma, E. Wang, X. Cai, H.-Z. Huang, X.-N. Wang, and Z.-Y. Zhu, "Quark matter 2006 conference," Journal of Physics G, vol. 34, 2006.

[4] W. Kittel and E. A. de Wolf, Soft Multihadron Dynamics, World Scientific, Singapore, 2005.

[5] A. Bialas and R. Peschanski, "Moments of rapidity distributions as a measure of short-range fluctuations in high-energy collisions," Nuclear Physics B, vol. 273, no. 3-4, pp. 703-718, 1986.

[6] A. Bialas and R. Peschanski, "Intermittency in multiparticle production at high energy," Nuclear Physics B, vol. 308, no. 4, pp. 857-867, 1988.

[7] R. C. Hwa, "Fractal measures in multiparticle production," Physical Review D, vol. 41, no. 5, pp. 1456-1462, 1990.

[8] F. Takagi, "Multifractal structure of multiplicity distributions in particle collisions at high energies," Physical Review Letters, vol. 72, pp. 32-35, 1994.

[9] M. I. Adamovich, M. M. Aggarwal, Y. A. Alexandrov et al., "On the jet-like and ring-like substructure in distributions of produced particles in central heavy-ion collisions at ultrarelativistic energies," Journal of Physics G, vol. 19, pp. 2035-2044, 1993.

[10] S. A. Bass, B. Müller, and D. K. Srivastava, "Parton rescattering and screening in $\mathrm{Au}+\mathrm{Au}$ collisions at RHIC," Physics Letters B, vol. 551, no. 3-4, pp. 277-283, 2003.

[11] L. Susskind, "Lattice fermions," Physical Review D, vol. 16, pp. 3931-3039, 1977.

[12] H. Kluberg-Stern, A. Morel, O. Napoly, and B. Petersson, "Flavours of lagrangian Susskind fermions," Nuclear Physics B, vol. 220, no. 4, pp. 447-470, 1983.

[13] I. M. Dremin, "Coherent hadron radiation at ultrahighenergies," Pisma v Zhurnal Eksperimentalnoi $i$ Teoreticheskoi Fiziki, vol. 30, pp. 152-156, 1979, English translation Journal of Experimental and Theoretical Physics Letters, vol. 30, pp. 140147, 1979.

[14] J. Hofmann, H. Stöcker, U. Heinz, W. Scheid, and W. Greiner, "Possibility of detecting density isomers in highdensity nuclear mach shock waves," Physical Review Letters, vol. 36, pp. 88-91, 1976.

[15] C. K. Chui, An Introduction to Wavelets, Academic Press, New York, NY, USA, 1992.

[16] D.-W. Huang, "Wavelet analysis in multiplicity fluctuations," Physical Review D, vol. 56, no. 7, pp. 3961-3969, 1997.

[17] I. M. Dremin, O. V. Ivanov, S. A. Kalinin, K. A. Kotelnikov, V. A. Nechitailo, and N. G. Polukhina, "Wavelet patterns in nucleusnucleus collisions at 158A GeV," Physics Letters B, vol. 499, no. 1-2, pp. 97-103, 2001.

[18] V. V. Uzhinsky, V. S. Navotny, G. A. Ososkov, A. Polanski, and M. M. Chernyavsky, "Wavelet analysis of angular distributions of secondary particles in high-energy nucleus-nucleus interactions: irregularity of particle pseudorapidity distributions," Physics of Atomic Nuclei, vol. 67, pp. 156-162, 2004.

[19] J. Fedorišin and S. Vokál, "Search for the righ-like structures in the emission of secondary particles in central 197Au collisions with emulsion nuclei at 11.6A GeV/c," JINR Preprint E1-2007-4, Dubna, Russia, 2007.

[20] J. Fedorisin and S. Vokal, "Wavelet analysis of angular spectra of relativistic particles in $208 \mathrm{~Pb}$ induced collisions with emulsion nuclei at 158A GeV/c," JINR Preprint E1-2007-66, Joint Institute for Nuclear Research (JINR) publishing department, Dubna, Russia, 2007, http://wwwl.jinr.ru/Preprints/prepr_2007_eng .html. 
[21] J. Fedorišin and S. Vokál, "Wavelet analysis of multiparticle correlations," FIZIKA B, vol. 17, no. 2, pp. 273-278, 2008.

[22] S. A. Bass, M. Belkacem, M. Bleicher et al., "Microscopic models for ultrarelativistic heavy ion collisions," Progress in Particle and Nuclear Physics, vol. 41, pp. 255-369, 1998.

[23] M. Bleicher, E. Zabrodin, C. Spieles et al., "Relativistic hadronhadron collisions in the ultra-relativistic quantum molecular dynamics model," Journal of Physics G, vol. 25, no. 9, pp. 18591896, 1999.

[24] O. V. Utyuzh, G. Wilk, and Z. Wlodarczyk, "Numerical modelling of Bose-Einstein correlations," Physics Letters B, vol. 522, no. 3-4, pp. 273-279, 2001.

[25] O. Utyuzh, G. Wilk, and Z. Wlodarczyk, "Modeling BoseEinstein correlations via elementary emitting cells," Physical Review D, vol. 75, Article ID 074030, 2007.

[26] M. Bystesrky, "Modeling Bose-Einstein correlation in heavy ion collision at RHIC," Nukleonika, vol. 49, supplement 2, pp. S37S39, 2004.

[27] C. F. Powell, P. H. Fowler, and D. H. Perkins, The Study of Elementary Particles by Photographic Method, Pergamon Press, Oxford, UK, 1959.

[28] W. H. Barkas, Nuclear Research Emulsions Vol. I and II, Academic Press, New York, NY, USA, 1963. 

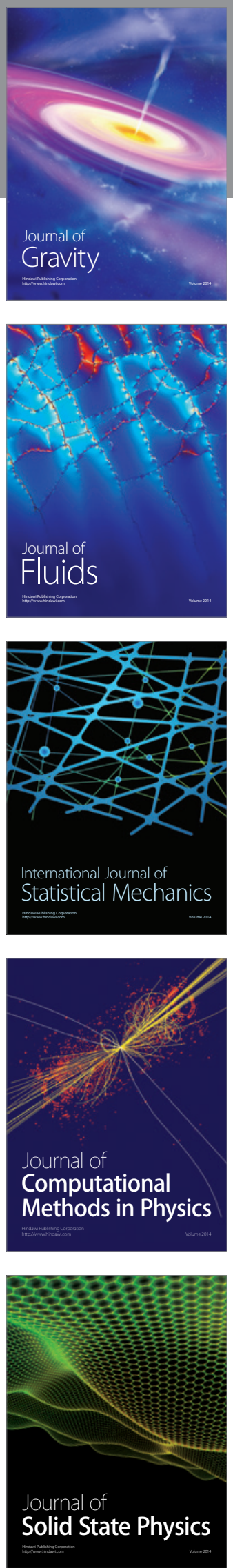

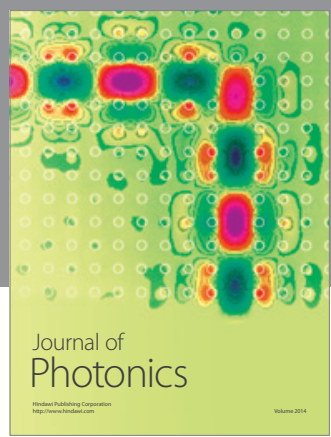

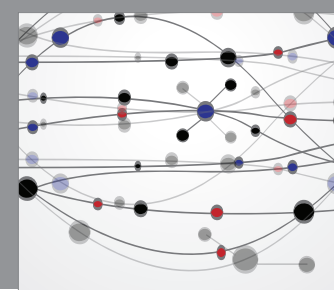

The Scientific World Journal

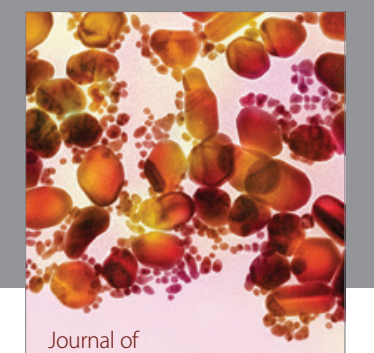

Soft Matter
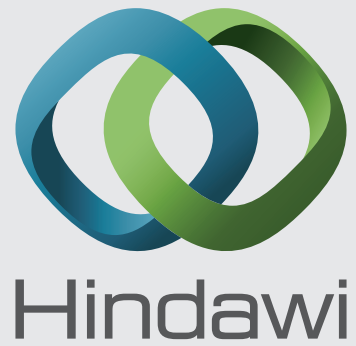

Submit your manuscripts at

http://www.hindawi.com
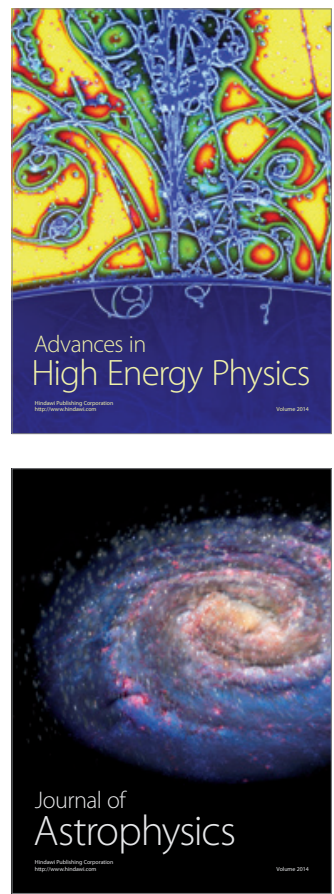
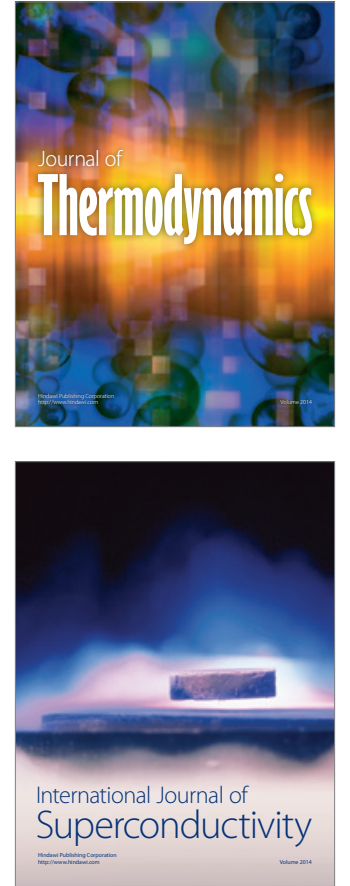
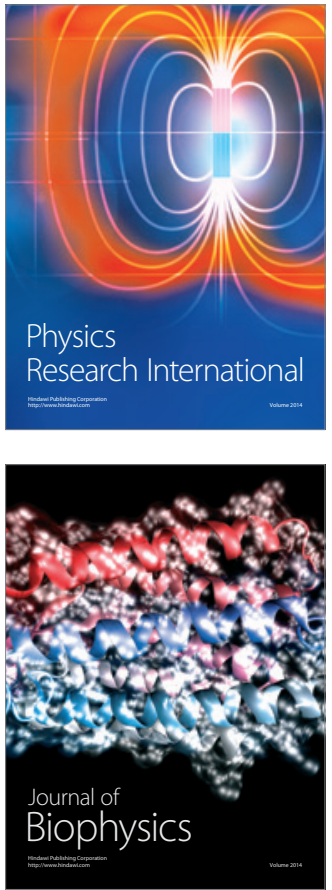
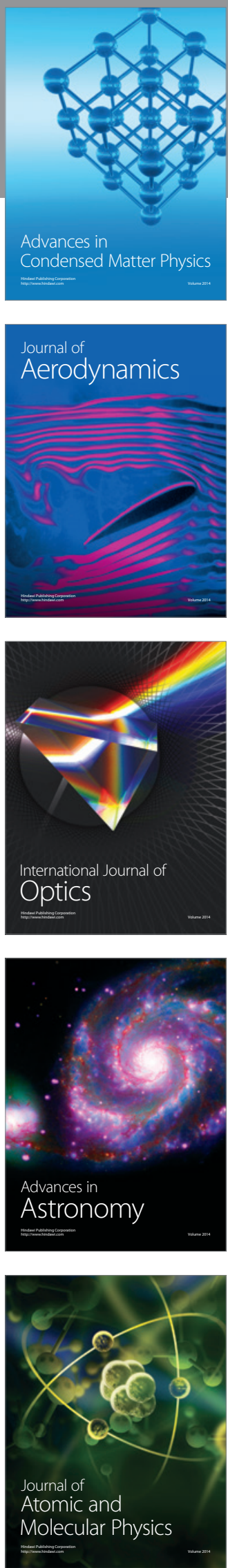